\title{
Penser les phénomènes de ludicisation à partir de Jacques Henriot
}

\section{Sébastien Genvo}

\section{OpenEdition}

\section{Journals}

Édition électronique

URL : http://journals.openedition.org/sdj/251

DOI : $10.4000 /$ sdj.251

ISSN : 2269-2657

Éditeur

Laboratoire EXPERICE - Centre de Recherche Interuniversitaire Expérience Ressources Culturelles Education

\section{Référence électronique}

Sébastien Genvo, "Penser les phénomènes de ludicisation à partir de Jacques Henriot », Sciences du jeu [En ligne], 1 | 2013, mis en ligne le 01 octobre 2013, consulté le 27 mars 2021. URL : http:// journals.openedition.org/sdj/251 ; DOI : https://doi.org/10.4000/sdj.251

Ce document a été généré automatiquement le 27 mars 2021.

\section{(c)}

La revue Sciences du jeu est mise à disposition selon les termes de la Licence Creative Commons Attribution - Pas d'Utilisation Commerciale - Pas de Modification 4.0 International. 


\title{
Penser les phénomènes de ludicisation à partir de Jacques Henriot
}

\author{
Sébastien Genvo
}

1 On pourrait penser que l'une des principales originalités des théories élaborées par Jacques Henriot est de considérer l'attitude ludique comme première dans l'apparition des phénomènes ludiques. Ceci n'a par exemple pas été le cas de nombreuses approches anglophones relevant des game studies qui reposaient avant tout sur une conception structuraliste (Genvo, 2008). Le temps semble à ce titre conforter le positionnement heuristique du philosophe : on peut remarquer qu'aujourd'hui plusieurs chercheurs à l'avant plan de ce champ anglophone revendiquent une prise en compte de l'activité du joueur, du «play», dans l'élaboration de leur cadre théorique (Taylor, 2009 ; Sicart, 2011), alors même que Jacques Henriot n'y est pas présent (faute de traduction). Si l'on peut considérer sur ce plan que Jacques Henriot est précurseur, il nous semble cependant que la principale force de sa pensée au regard des problématiques actuelles du champ se situe à présent à un autre niveau (qui découle cependant de son positionnement heuristique initial).

2 La fortune de la notion de "gamification» dans les pratiques professionnelles, la multiplication des serious games, l'avènement du « newsgaming », en somme la contagion du jeu à de nombreuses sphères d'activité qui lui étaient autrefois étrangères, donne une actualité nouvelle à la réflexion suivante du philosophe :

Le monde où je vis est un monde où il est de plus en plus question de jeu : non seulement parce qu'il me semble que l'on y joue chaque jour davantage, mais surtout parce que l'idée même du jeu s'applique constamment à de nouvelles situations, à des formes de conduite et de pensée auxquelles il eut paru, récemment encore, inconvenant de l'appliquer (Henriot, 1989, p. 27).

En mettant l'emphase sur le jeu comme idée susceptible de se transformer à travers les époques et les lieux, Jacques Henriot pose selon nous les fondements d'une pensée du jeu permettant de comprendre et d'analyser ce phénomène comme un processus mouvant et changeant. Ceci est un nouveau défi posé aux théorisations du jeu qui 
s'étaient construites autour du modèle «canonique du jeu » (Schmoll, 2010, p. 31), tel que défini par Johan Huinzinga puis Roger Caillois (ce type d'ancrage théorique étant majoritaire dans les game studies) : le jeu s'y définit à partir d'une suite de critères intrinsèques (le jeu est gratuit, libre, séparé du quotidien, etc.). La multiplication et la mutation des phénomènes ludiques montrent que ces définitions sont elles aussi des constructions culturelles reflétant avant tout une certaine idée de ce qu'est cette « chose nommée jeu » (Henriot, 1989). Jacques Henriot cherche à ce titre à s'écarter de ce genre de définition qu'il qualifie de « descriptives » (Henriot, 1989, p. 168). Il y aurait un invariant anthropologique de jeu chez les hommes (l'attitude ludique), mais ceux-ci se feraient cependant des idées distinctes de ce même phénomène (Henriot, 1989, pp. 83-84), la conception du jeu et les objets qui renvoient à cette activité étant toujours susceptibles d'évoluer.

Pour qu'une structure ludique qui n'ose pas encore dire son nom puisse faire l'objet d'une approche et d'une appréhension conceptuelle qui conduise à lui attribuer ce nom, il faut et il suffit que l'on adopte envers elle l'attitude que l'on adopte envers ce que l'on appelle habituellement un jeu. Ce qui s'atténue au point de disparaître parfois quand on passe d'un objet à l'autre, c'est l'ensemble des connotations traditionnellement attachées au terme de jeu (Henriot, 1989, p. 45).

De façon à comprendre les phénomènes de contagion et de mutation du jeu, il est donc nécessaire de ne pas tenir pour inhérentes les caractéristiques et la dimension ludique d'un objet, mais au contraire de les questionner pour décrire comment certains objets qui n'étaient pas encore considérés comme des jeux en viennent progressivement à être qualifiés de la sorte et comment, ce faisant, les conceptions que l'on se faisait de ce qu'est un jeu sont amenées à changer. Nous proposons de nommer ces processus de contagion et de transformation du jeu des processus de ludicisation.

Le positionnement adopté par Henriot est encore aujourd'hui novateur dans le paysage des études sur le jeu, peu de théories essayant de le penser comme un processus en perpétuelle transformation qu'il faut comprendre en dehors de toute connotation fixée a priori. Sur ce point, Thomas Malaby esquisse un cadre théorique équivalent dans un article datant de 2007 qu'il intitule de façon assez signifiante: «Beyond play. A new approach of games » (il ne fait pas cependant mention de Jacques Henriot). Plusieurs pistes esquissées par Henriot sur cette approche des jeux comme idées mouvantes restent selon nous encore aujourd'hui largement inexploitées alors même que leur exploration permet de construire un cadre théorique permettant de rendre compte des processus de transformation du jeu, des idées qui s'y réfèrent, mais aussi d'en faire l'historique, et ainsi d'aborder un terrain de recherche encore en friche. La vocation du présent article est donc de construire, en partant des réflexions de Jacques Henriot, un cadre analytique des processus de ludicisation, qui donnera les outils nécessaires pour en retracer les évolutions actuelles et passées. En somme, il s'agit de réactualiser certaines réflexions de Jacques Henriot et les poursuivre au regard des défis ludologiques actuels. Comme nous le verrons, les jeux sont avant tout des agencements socio-culturels et le rôle du ludologue est de comprendre et décrire les processus de formation et d'évolution de ces agencements, à l'aide d'un ensemble d'outils théoriques forgés à cet égard. 


\section{Un agencement créé par l'attitude ludique}

6 Puisque selon Henriot, l'attitude ludique est première dans l'apparition des phénomènes ludiques, dans ce qui fait qu'il y a jeu, il faut tout d'abord revenir sur certains aspects de la définition de cette attitude pour établir notre cadre théorique. L'un des termes centraux employés par Henriot pour la qualifier est de la désigner comme un "procès métaphorique » (Henriot, 1989, p. 300), de «l'imaginaire en acte » (Henriot, 1989, p. 155). Cette assertion rejoint le point de vue de Jean-Marie Schaeffer (1999, p. 234) qui définit le jeu comme "compétence intentionnelle complexe» fictionnelle, acquise à un âge très précoce, qui tire profit d'une structuration mentale précablée, caractérisée par le fait que le joueur fait comme si ce qu'il faisait était autre. Si le joueur d'échecs s'applique à déplacer minutieusement chacune de ses pièces en envisageant le poids que chacune de ses décisions prendra dans la suite du jeu, son geste ne saurait par exemple se résumer à cette seule action pour qu'il puisse effectivement y avoir jeu. Comme le disent les amateurs de jeu d'échecs, il ne s'agit pas uniquement de "pousser du bois ». Pour entrer véritablement dans le jeu, le joueur doit faire comme si le plateau et les pièces qui se présentent à lui ne se résumaient pas uniquement à ce qu'ils sont, mais sans prendre pour autant le simulacre pour la réalité ; il doit entrer dans un univers de sens singulier : il va transposer les choses du monde où il vit dans un ordre nouveau, régi à la fois par des règles de la vie courante et par des règles spécifiques qui n'y ont pas habituellement cours. Mais le joueur ne va pas non plus totalement s'abstraire de la " réalité ordinaire » : jouer ce n'est pas rêver (Henriot, 1989, p. 221). Comme nous l'avons montré par ailleurs (Genvo, 2009), la notion d'aire intermédiaire d'expérience de Donald $\mathrm{W}$. Winnicott semble tout à fait appropriée pour qualifier cette réalité où se déroule le jeu : "Cette aire où l'on joue n'est pas la réalité psychique interne. Elle est en dehors de l'individu, mais elle n'appartient pas non plus au monde extérieur » (Winnicott, 1975, p. 67). Si la pensée du joueur s'absente dans une aire intermédiaire, l'action du joueur sur le monde est donc tout aussi effective que celle issue d'une autre activité. Elle va donc également s'inscrire dans un certain contexte pragmatique qui lui donne forme. Le jeu n'est pas sans relation avec les dispositifs techniques qui lui donnent forme, son époque, le milieu, "comme serait celui d'un dieu s'il lui prenait la fantaisie de jouer. Après tout, l'Enfant divin d'Héraclite, s'il joue, n'est-ce pas, dans les siècles des siècles, à un seul et même jeu : cette petteia à laquelle, précisément, jouaient les contemporains d'Héraclite?» (Henriot, 1989, p. 226).

7 En affirmant que "jouer c'est faire », Henriot affirme aussi qu'adopter une attitude ludique revient à adopter un ensemble de règles qui structurent le comportement (Henriot, 1989, p. 227), que ces règles aient ou non été préalablement formalisées ; jouer comporte un ensemble de règles formelles et informelles, voire implicites (cf. Salen \& Zimmerman, 2004). Jouer à faire l'avion ce n'est pas jouer à faire le train. Dans tous les cas, selon Henriot, les règles sont l'équivalent de fins et leur ensemble va constituer la thématique du jeu.

Si la fillette s'oblige à ce que les fougères ne la touchent pas, ce n'est pas afin de parvenir à l'autre extrémité du jardin. Le but qu'elle se fixe est justement celui-là : traverser le jardin sans que les fougères ne la touchent. La règle fait corps avec le but. [...] Dans un cas semblable, il est impossible de dissocier la règle de la fin poursuivie, autrement dit la règle du jeu... du jeu lui-même (Henriot, 1989, p. 227). 
Enfin, il est aussi important de préciser que le joueur ne se fixe pas uniquement des buts, il agence pour y parvenir un certain nombre de moyens.

Tout acte de jouer se présente comme une structure opératoire de forme diachronique. Il consiste à œuvrer en vue de la réalisation d'un dessein défini, par l'agencement et la mise en œuvre plus ou moins coordonné de moyens (Henriot, 1989, p. 221).

9 Prenant l'exemple de la guerre, Henriot fait ainsi la distinction entre la fin (soumettre l'ennemi) et les moyens (la violence). La particularité des moyens mis en œuvre par le joueur est qu'ils ne peuvent lui garantir une efficacité assurée. Pour Henriot, jouer c'est faire l'exercice du possible, sinon on ne joue pas. « Le propre du jeu est de s'élaborer, de se poursuivre, d'aller jusqu'à son point d'achèvement dans un climat de constante incertitude » (Henriot, 1989, p. 236). Il est important de préciser que cette incertitude n'est pas simplement le fait de la situation dans laquelle se trouve le joueur mais est avant tout relative à l'individu, l'imprévisibilité dépendant des connaissances du joueur, de sa position dans le jeu :

l'incertitude est le fait de quelqu'un. L'augmentation du nombre et de la richesse des informations tend à la réduire et permet une meilleure prévision. Mais le joueur ne prend ni le temps ni la peine de s'informer suffisamment. [...] Qu'il gagne ou qu'il perde il aura toujours l'impression que les choses auraient pu tourner différemment (Henriot, 1989, pp. 237-238).

10 En somme, nous pouvons donc avancer que lorsqu'il adopte une attitude ludique, un individu procède à l'agencement d'un ensemble de règles et de moyens (qui structurent son attitude ludique), d'un monde fictionnel (puisque jouer c'est « faire comme si ») et d'un contexte pragmatique (le monde extérieur dans lequel s'incarne le jeu). Il nous semble important de souligner dans cette précédente définition le terme d'agencement, qui est aussi employé à plusieurs reprises par Henriot. Comme l'indique Deleuze, un agencement est « une multiplicité qui comporte beaucoup de termes hétérogènes, et qui établit des liaisons, des relations entre eux [...]. Aussi la seule unité de l'agencement est de co-fonctionnement» (Deleuze et Parnet, 1977, p. 84). On doit donc porter attention aux relations qui s'effectuent entre les différentes sphères du jeu, comme elles peuvent être représentées dans le schéma suivant : 
Schéma 1. Un agencement de jeu créé par l'attitude ludique

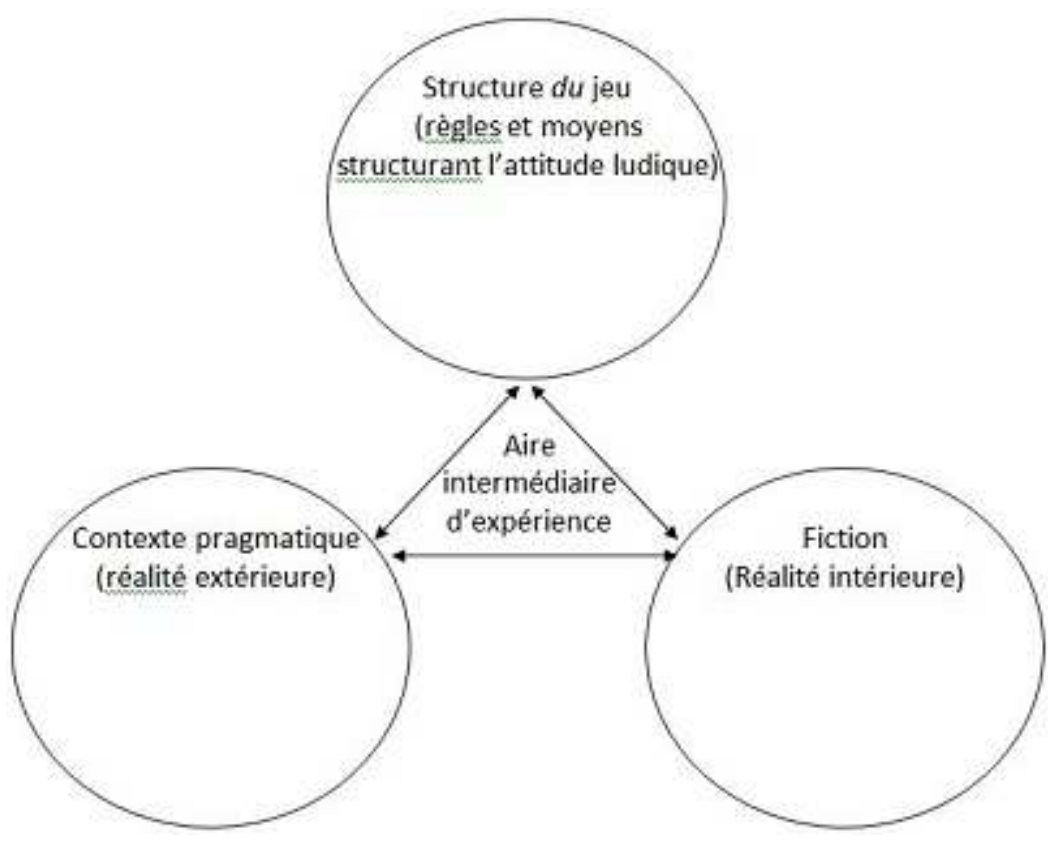

11 Analyser les agencements produits par l'acte ludique c'est à la fois comprendre comment les règles prennent forme dans un contexte pragmatique donné (qui va aussi avoir une incidence sur leur formulation), comment ce contexte fournit un certain support de fiction (l'attitude ludique oscillant entre réalité intérieure et extérieure, entre engagement et distanciation [Henriot, 1989, p. 260]), et comment cette fiction est régie par des règles dont elle permet conjointement l'appréhension (l'utilisation du médiéval fantastique est par exemple très commode dans les jeux vidéo en ce qu'il permet d'identifier facilement qui sera l'ennemi ou encore qui peut apporter son soutien, en jouant sur les références intertextuelles).

\section{Contenus et expressions des agencements de jeu}

Selon Jacques Henriot, même si le jeu n'apparaît véritablement qu'au moment où quelqu'un en adopte l'intention, il est indéniable que «tout le monde sait qu'il y a des objets conçus, fabriqués, vendus pour servir d'outils de jeu» (1989, p. 101). Une précision doit dans ce cadre être apportée en faisant la distinction entre 1) les structures de jeu, qui sont les objets destinés à servir d'outils de jeu et qui comportent notamment un système de règles formalisées pouvant faire l'objet d'une « représentation publique » indépendante du joueur (l'équivalent en anglais du terme "rules of games ») et 2) la structure $d u$ jeu ("rules of play»), qui est l'ensemble des schèmes, des règles informelles et formelles que suivent les joueurs lorsqu'ils jouent à un jeu et qui peuvent différer de celles qui ont été préalablement établies par le jeu (les joueurs peuvent très bien ajouter des règles informelles à un jeu, voire en modifier les règles durant la pratique et transformer la structure de jeu). La conception d'un jeu comporte alors une dimension fondamentalement communicationnelle; elle implique de comprendre les modalités de transmission d'une signification partagée de jeu pour inciter à adopter une attitude ludique. Une structure de jeu fait appel à certaines 
connaissances ludiques d'un utilisateur cible, à travers des marqueurs pragmatiques qui ont pour vocation d'être signifiants pour eux en terme de jeu (play).

Ce qui fait l'essence de tout jeu, pourrait-on dire en généralisant, ce n'est, ni sa valeur en tant que divertissement, ni son détachement par rapport à ce que l'on appelle la vie réelle : c'est le fait que la situation à laquelle il donne sens offre un nombre déterminé de caractères objectivement repérables, définissables et analysables (Henriot, 1989, p. 218).

Il est donc nécessaire de décrire de quelle façon les objets qui revendiquent être des jeux peuvent s'inscrire à certains égards dans des dimensions que l'on confère usuellement à l'activité pour signifier que "ceci est un jeu» (Bateson, 1977). Cela n'exclut pas la possibilité de trouver dans ces mêmes objets d'autres caractéristiques qui vont à l'encontre des canons établis du jeu. Ce dernier aspect peut en effet amener le terme de jeu à s'appliquer à des réalités nouvelles. Puisqu'il est envisageable, comme le montre Bogost (2007), de tisser des liens entre rhétorique et jeu vidéo, nous considérerons que ces attributs qui ont pour vocation de faire œuvre de médiation ludique constituent ce que nous nommons l'éthos ludique de l'œuvre. Selon Nicole Pignier (2008, p. 52), le concept d'éthos, issu de la rhétorique classique, désignait les traits de caractère que l'orateur devait montrer à l'auditoire pour donner de l'autorité à ce qu'il disait et garantir son discours. Jean-Jacques Boutaud (2008) précise que si l'éthos renvoie au travail de l'énonciateur pour construire son monde propre et le faire partager, il faut aussi prendre en compte dans le cadre des TICs qu'il s'agit de construire un univers où l'utilisateur peut se retrouver et évoluer en connivence. L'éthos serait alors à comprendre comme une notion porteuse d'un système de valeurs. Dans notre cas, cela incite à analyser les valeurs conférées à l'activité ludique par un logiciel pour être actualisé comme jeu. Dans leur ouvrage, Digital Play, Kline, DyerWitheford et De Peuter (2003) identifient par exemple dans les premiers moments de l'histoire des jeux vidéo une prédominance de thématiques liées à ce qu'ils nomment la "masculinité militarisée ». Ces thématiques ont permis l'essor économique du jeu vidéo du fait de son public cible prioritaire, les adolescents masculins, et de son contexte d'usage initial, les bars et salles d'arcade, qui servaient aussi de terrain d'expérimentation pour développer les jeux sur les premières consoles de salon.

Pour comprendre la façon dont une situation peut être sujette au jeu, il faut également préciser que le jeu ne peut prendre place en toute situation. Par exemple une inondation ne pourrait s'y prêter :

Si l'on se retient de le dire c'est un peu par crainte de heurter l'opinion, de blesser les sensibilités; mais c'est avant tout parce que les situations que créent de tels événements ne laissent pratiquement aucune place à l'initiative de ceux qui s'y trouvent pris. Ils n'ont pas le choix. Très exactement, ils n'y peuvent rien (Henriot, 1989, p. 193).

En somme, certaines situations présentent des caractéristiques qui ne permettent pas aux personnes qui y sont impliquées d'adopter une attitude ludique à leur égard, le fait de ne pas pouvoir mener une action qui ait une répercussion signifiante sur la suite des événements étant l'un de ces aspects (le joueur ne pourra faire l'exercice des possibles dans ce cas précis). « Il faut que le jeu soit joué, mais pour qu'il soit joué, il faut d'abord qu'il soit jouable » (Henriot, 1989, p. 108). Le rapport entre attitude ludique et structure de jeu est donc véritablement à comprendre comme un co-fonctionnement. Si certaines situations ne permettent pas à l'individu d'adopter une attitude ludique, d'autres, en revanche, présentent alors des traits qui rendent son adoption plus aisée, elles 
présenteront une plus grande " jouabilité »: «Je me propose de théoriser sous le nom de jouabilité ce qui, sur le plan purement structural, fait d'une situation un jeu potentiel » (Henriot, 1989, p. 217). Par exemple, pour un enfant, il est usuellement plus difficile de jouer durant les cours, alors que la récréation est un moment propice au jeu. Cela ne veut pas dire qu'il est impossible de jouer en classe, mais que la structure de la seconde situation (son système de règles et de contraintes) comporte une jouabilité plus élevée que la première; nous avons notamment avancé par ailleurs (Genvo, 2011) que la jouabilité d'une situation pouvait s'analyser à partir des degrés de contingence qu'elle permet. Il faut à nouveau souligner que la jouabilité d'une situation - son adaptation à une attitude ludique - dépendra de l'individu qui l'actualise. Une même situation peut très bien présenter un caractère de jouabilité pour un individu tout en étant injouable pour un autre. Par exemple, le faible nombre de tours et la réduction rapide des possibilités font que l'exercice du possible peut être rapidement rendu caduc dans le jeu du morpion. En effet, un joueur expérimenté peut facilement gagner ou forcer le nul sans jamais perdre, pourvu qu'il suive un certain nombre de règles logiques. Et si les deux joueurs suivent scrupuleusement ces règles, la partie se terminera automatiquement par un nul, éliminant toute part de contingence. Le jeu du morpion risque donc de ne pas être jouable dans ce cas.

Ce faisant, en comportant une jouabilité et un éthos donné, une structure de jeu va aussi s'adresser à certains types de joueurs, qui sont susceptibles de disposer ou d'acquérir certaines connaissances et "compétences ludiques» (Duflo, 1997). Elle dessine en somme un certain « joueur-modèle », qui n'est pas nécessairement le joueur empirique mais qui est en tout cas la stratégie interprétative que met en forme la structure de jeu pour constituer l'ensemble de ses interprétations légitimables. Le joueur-modèle témoigne du contexte pragmatique pour lequel la structure de jeu a été initialement conçue, ce qui n'empêche aucunement les réappropriations. Comprendre le rôle du contexte initial dans la mise en forme d'un joueur-modèle et l'itinéraire des appropriations successives de la structure - de même que la complémentarité ou résistance qu'elle offre à ses appropriations - peut être le rôle d'une socio-histoire des jeux vidéo qui reste à faire. De nombreux jeux vidéo, tels que la série des Sim City (Maxis, 1989 - 2011), sont par exemple aujourd'hui employés en salle de classe alors que ce n'était pas leur vocation première et qu'ils présentent certaines caractéristiques qui peuvent offrir de la résistance à ces nouveaux contextes, par exemple le temps d'une partie. Il est d'ailleurs possible qu'une même structure de jeu construise plusieurs joueurs-modèles. Nous avons par exemple montré (Genvo, 2009) comment la structure de World of warcraft (Blizzard, 2004) construisait conjointement un joueur-modèle pour le joueur néophyte et un autre joueur-modèle pour les joueurs expérimentés, ceci afin de diversifier le type de public usuel du jeu massivement multijoueur en ligne, jusqu'alors réservé à des joueurs initiés.

17 Le schéma que nous avons proposé peut donc être modifié de la façon suivante de sorte à prendre en compte l'ensemble de ces considérations : 


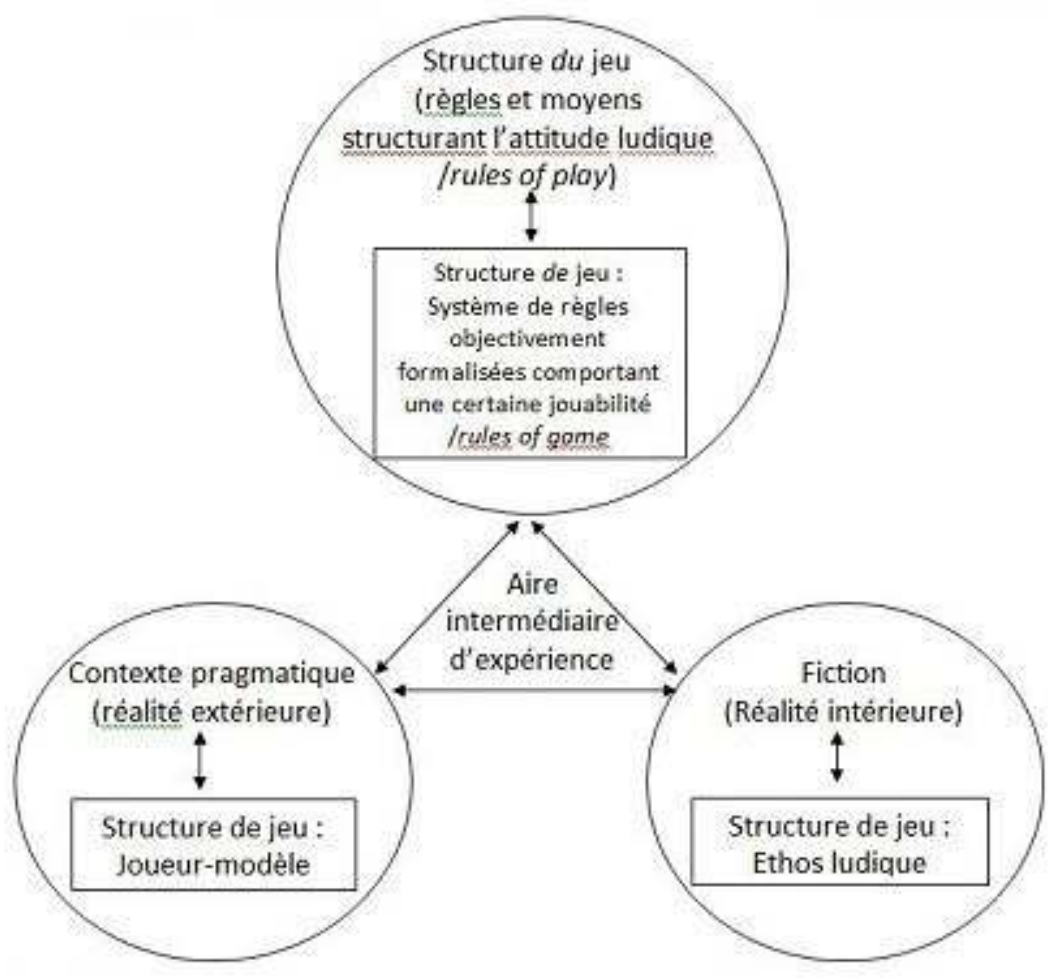

Dans le cadre des agencements de jeu, comme dans tout agencement (Deleuze et Guattari, 1980), on peut faire la distinction entre le plan du contenu (la structure de jeu, le game) et le plan de l'expression (le play), les deux étant en présupposition réciproque : le joueur-modèle s'inscrit dans un contexte pragmatique, mais le joueurmodèle invite aussi à produire son contexte à travers une certaine stratégie interprétative analysable; l'éthos ludique puise dans des référents fictionnels pour constituer un système de valeur, qui aura vocation à persuader son destinataire à adopter une attitude ludique à travers les représentations véhiculées; les règles de la structure de jeu comportent une certaine jouabilité qui structurera en partie l'attitude ludique, celle-ci pouvant amener des modifications dans les règles, que ce soit en ajoutant des règles informelles ou en faisant évoluer la structure de jeu elle-même. Il est important de préciser que l'acte de jouer, qui procède par «agencement de moyens", s'inscrit de fait dans un territoire qui fait la singularité de chaque agencement : « tout agencement est d'abord territorial, la première règle concrète des agencements c'est de découvrir la territorialité qu'ils enveloppent, le territoire fait l'agencement » (Deleuze et Guattari, 1980, p. 629). Le territoire du jeu, c'est bien sûr l'aire intermédiaire d'expérience qui fait le lien entre les trois sphères décrites dans le schéma. "Le territoire est fait de fragments décodés de toutes sortes, empruntés aux milieux, mais qui acquièrent alors une valeur de propriétés » (Deleuze et Guattari, 1980, p. 629). Analyser les territoires de jeu, c'est donc comprendre comment les termes mis en avant dans ce schéma forment, à travers leurs frictions et complémentarités, un agencement particulier.

19 Spacewar, ${ }^{1}$ tel qu'il a été développé par Steve Russel, était le produit d'un certain lieu, le MIT, développé pour des étudiants ingénieurs qui disposaient de certaines compétences ludiques pour l'actualiser, qui partageaient certains référents fictionnels communs (dont une fascination pour la science fiction), de même que certaines valeurs accordées 
à l'activité ludique. Il s'inscrivait dans une pratique de hacking qui prônait, entre autres, la libre circulation de l'information et l'ouverture des objets aux modifications apportées par ses utilisateurs. L'ensemble de ces éléments se retrouve dans les propriétés de la structure de jeu de Spacewar, c'est-à-dire dans son éthos ludique, dans sa jouabilité et dans le joueur-modèle mis en forme. Computer Space, la version commerciale du précédent jeu développé par Bushnell à destination des bars et des salles d'arcade, s'inscrivait quant à lui dans un territoire différent et formait de ce fait un agencement tout autre. Bushnell modifia la structure du jeu pour y faire correspondre un modèle économique, celui du monnayeur des bornes d'arcade. Même si les référents fictionnels restaient les mêmes, les valeurs conférées à l'activité étaient dès lors toutes autres que celles des hackers qui avaient donné naissance à Spacewar. Comme le montrent Kline, Dyer-Whiteford et De Peuter (2003, p. 56), la marchandisation du jeu entraîne des logiques de restriction d'usages, de normalisation qui rentrent en friction avec les promesses d'exploration libre et ludique qui étaient aussi à l'origine du domaine, la communauté hacker ayant largement contribué à sa naissance. Le joueur-modèle de Computer Space ne fut cependant pas modifié et adapté à son nouveau contexte pragmatique (celui des arcades), les compétences ludiques requises étant toujours celles des étudiants ingénieurs des instituts de technologie, ce qui entraîna l'échec de Computer Space. Cet exemple montre conjointement la nécessité de penser la structure de jeu non pas comme une forme immuable, mais bien comme le résultat d'un processus de mutations, de transformations. La structure de jeu est une forme toujours susceptible de changer et qui peut envahir de nouveaux territoires. Cela contribue par là-même à modifier la signification que l'on attribuait à cette activité et à élargir l'idée de jeu à d'autres réalités, ce que montre l'exemple des processus de ludicisation qui traversent l'histoire de Space War puis Computer Space.

L'agencement se divise aussi d'après un autre axe. Sa territorialité (contenu et expression compris) n'est qu'un premier aspect, l'autre aspect étant constitué par les lignes de déterritorialisation qui le traversent et l'emportent (Deleuze, 1986, p. 630).

20 À ce titre, la contagion actuelle du jeu à des domaines qui lui étaient étrangers et l'équivalence qu'établit Jacques Henriot entre jeu et idée encouragent à faire un lien avec la théorie d'épidémiologie des représentations développées par Dan Sperber :

Les idées non seulement peuvent se transmettre, mais même, en étant transmises à nouveau par ceux qui les reçoivent, elles peuvent, de proche en proche, se propager. [...] Expliquer la culture, c'est expliquer pourquoi et comment certaines idées sont contagieuses. Il faut pour cela développer une véritable épidémiologie des représentations (Sperber, 1996, p. 8).

21 Dan Sperber précise à ce titre qu'expliquer le caractère culturel de certaines représentations, c'est répondre à la question suivante : pourquoi ces représentations sont-elles plus contagieuses et réussissent-elles mieux que d'autres dans une population humaine donnée ? Pour y répondre dans le cas des jeux, il faut prendre en considération les processus de ludicisation qui traversent la formation d'agencements singuliers.

\section{Diagramme des mécanismes de ludicisation}

Comme l'indique Henriot, les jeux sont des phénomènes sujets aux variations, ces variations dépendant du temps et de l'espace (Henriot, 1989, p. 15). Pour Deleuze et 
Parnet, les vecteurs de mutation, de variation, sont une des composantes de tout agencement, qui l'orientent vers un devenir :

Pas d'agencement sans territoire, territorialité, et re-territorialisation qui comprend toutes sortes d'artifices. Mais pas d'agencement non plus sans pointe de déterritorialisation, sans ligne de fuite, qui l'entraîne à de nouvelles créations, ou bien vers la mort? » (Deleuze et Parnet, 1977, p. 87).

Ces variations, ces pointes de déterritorialisation, tracent alors un diagramme, qui est pour Deleuze une notion complémentaire et indissociable de celle d'agencement. Le diagramme est une potentialité de faits, la cause abstraite, des agencements concrets, qui en sont les effets. Deleuze définit la notion de diagramme en se référant à Foucault, qui l'avait employée pour définir, dans Surveiller et Punir, le Panopticon, architecture carcérale permettant au surveillant de tout voir sans être vu et aux détenus d'être vus sans rien voir.

Le Panopticon ne doit pas être compris comme un édifice onirique : c'est le diagramme d'un mécanisme de pouvoir ramené à sa forme idéale ; son fonctionnement, abstrait de tout obstacle, résistance ou frottement, peut bien être représenté comme un pur système architectural et optique (Foucault, 1975, p. 233).

Dans l'optique de Deleuze, le diagramme décrit par Foucault est une «machine abstraite ", informelle, elle fait abstraction de ses fonctions et matières, tandis que les agencements concrets procèdent à une « actualisation-intégration » sur des substances et des fonctions, sur des formes de contenu et des formes d'expression (Deleuze, 1986, pp. 45- 46) : le Panopticon en tant que diagramme peut s'intégrer et s'actualiser dans les écoles (la fonction est alors d'éduquer, les substances sont les élèves), les usines, les hôpitaux, les prisons, etc.

De la même façon, les différentes formes de jeu témoignent de diagrammes de ludicisation dont les mécanismes s'actualisent dans différentes fonctions et différentes formes du jeu (ce qui témoigne du caractère mouvant des idées du jeu) : le jeu peut être employé pour divertir, pour informer, pour éduquer, etc. Il prend forme dans la cour d'école, dans le salon, dans les laboratoires d'institut de technologie, etc. Décrire les diagrammes de ludicisation c'est rendre compte des mécanismes qui président à la mise en forme d'agencements de jeu singuliers. La notion de genre dans les jeux vidéo, et son rôle dans les pratiques, montre de quelle façon les agencements de jeu sont traversés par un diagramme abstrait, qui les préside et construit à travers eux un réel à venir, des points de potentialité. Une structure de jeu propose souvent par exemple de perfectionner le fonctionnement de mécanismes déjà éprouvés tout en apportant certaines variations au système d'origine. C'est le cas d'un jeu comme Half-Life (Sierra, 1998) qui reprenait les principaux mécanismes des jeux s'apparentant à Doom (Id Software, 1993) tout en les complexifiant. Il arrive aussi qu'une structure de jeu propose par son système une majorité de modalités d'action véritablement inédites. Certaines de ces modalités acquièrent par la suite le statut de canons et servent de briques de base à la structure d'autres jeux, afin que ces derniers soient aussi reconnus comme potentiellement ludiques. On a par exemple pendant un temps parlé de «Doomlike » pour parler du jeu s'apparentant à la production d'Id Software. On constate dans ces deux cas, que ce qui opère au centre des mécanismes de ludicisation, ramené à sa «forme idéale », «son fonctionnement, abstrait de tout obstacle » (Foucault, 1975, p. 233), c'est un pouvoir-faire proposé à l'individu, une modalité d'action qui va ensuite s'incarner et s'actualiser dans un agencement de jeu. 
Le genre de jeu vidéo renvoie à ce qu'il y a de plus important pour le joueur : la façon de jouer. Si l'on se penche rapidement sur quelques-uns des termes employés couramment pour classer les jeux, on retrouve en effet de façon très marquée les activités du joueur: jeu de plates-formes (il s'agit de sauter d'une plate-forme à l'autre), jeu de tir (il s'agit de tirer sur des monstres), jeu de rôle (il s'agit de se concentrer sur son personnage - persona - pour le faire progresser), jeu d'aventure (adventura, ce qui advient, dit bien le principe du jeu, où il s'agit de faire advenir des événements, du récit, en résolvant des énigmes) (Letourneux, 2006, p. 40). de jouer ne donne pas par avance sa fonction, sa valeur. Pour une même façon de jouer, il peut y avoir une multiplicité d'agencements, de fonctions (divertir, éduquer, informer,...), de règles qui donnent forme à ce mécanisme, de fictions qui viennent l'habiller, de valeurs conférées, de joueurs-modèles, etc. Il est cependant important de lever une possible ambigüité. Le genre vidéoludique n'est pas un diagramme car il résulte lui-même de plusieurs actualisations d'un diagramme. Il est lui aussi l'effet de mécanismes de ludicisation. Comme l'indique Deleuze, "l'actualisation est une intégration, un ensemble d'intégrations progressives, d'abord locales, puis globales ou tendant à être globales, opérant un alignement, une homogénéisation, une sommation des rapports de force » (Deleuze, 1986, p. 45). Certains agencements vont alors, dans un contexte donné, paraître plus typiques que d'autres de ce qu'on pense être un jeu (cette perception pouvant évoluer). Il peut y avoir des périodes de stabilisation des variations. Dans cette perspective, chaque agencement effectue un diagramme à différents degrés : «c'est comme des coefficients d'effectuation, et plus le degré est haut, plus l'agencement diffuse dans les autres, adéquat à tout le champ social » (Deleuze, 1986, p. 48). l'ordinateur individuel, à son acceptation en tant que possible outil de jeu, offre un terrain de recherche particulièrement riche pour rendre compte de différentes actualisations d'un diagramme de ludicisation et de la façon dont un agencement particulier a diffusé dans d'autres agencements, en partant du local vers le global. L'un des titres fondateurs du jeu d'aventure se nomme justement Adventure (1976), et cette homonymie ainsi que la pérennité de cette dénomination ne sont pas anodines. En effet, à l'époque de son apparition, Adventure présente un agencement original de mécanismes de jeux qui servit de socle commun à une multitude d'autres jeux et qui lui furent par la suite apparentés : l'exploration d'un monde fictionnel, la présence d'un récit dont l'avènement dépend de la progression du joueur, la présence d'énigmes dont la résolution conditionne l'avancée du joueur. Comme on peut le constater, une même logique unit ces trois mécanismes : celle de l'exploration. Dans Adventure, celle-ci se fait à travers des textes de description adressés au joueur, qui peut entrer des instructions simples pour interagir avec l'environnement (« go north », « take bottle »). Le contexte de développement permet de comprendre en grande partie ce qui guida l'adoption de ces choix de conception, de même que leur fortune pour guider la conception d'autres jeux de l'époque. Adventure a été initialement développé par William Crowther à partir de 1975. Crowther est notamment membre d'une équipe de programmation dans une entreprise technologique de recherche et développement du Massachusetts, Bolt, Beranek and Newman (BBN). Il participa, entre autres, au développement du réseau Arpanet (précurseur d'Internet), qui fut essentiellement utilisé par les chercheurs de firmes et d'universités, et par les militaires. Mais Crowther avait aussi plusieurs hobbies qui expliquent les thématiques au centre du jeu qu'il développa. Il était en effet un 
spéléologue expérimenté et joueur de Dungeons \& Dragons. Le logiciel que Crowther commence à développer en 1975 propose d'explorer un système qui reproduit la topographie de grottes dans le Kentucky que Crowther avait réellement explorées et cartographiées (ce que le joueur devra aussi faire dans Adventure s'il veut se repérer). Il y ajouta des trésors et quelques éléments de fantasy.

Les premiers utilisateurs de cette version d'Adventure furent les filles de Crowther et quelques collègues. Mais les joueurs furent rapidement plus nombreux que ce cercle restreint. En 1976, Don Wood, un jeune hacker, étudiant à l'université de Stanford, met la main sur une copie du jeu. Il renforça notamment les références faites à la fantasy, chères à la communauté hacker (en se rapprochant encore davantage de l'univers de Tolkien). Dans cette version, le jeu fit très vite le tour des machines connectées à Arpanet. La force du jeu de Crowther et Wood provient de l'agencement original de plusieurs mécanismes d'autres jeux l'ayant précédé et de son éthos ludique. Cet agencement fait particulièrement sens auprès de son public, essentiellement les chercheurs, étudiants, programmeurs appartenant à la communauté des hackers présente dans les campus américains connectés à Arpanet. Outre les références faites à Tolkien, l'exploration du réseau de cavernes par l'utilisation d'un langage naturel produisait un plaisir proche de la programmation (Montfort, 2003), ce qui généra de nombreuses vocations de game designer auprès d'un public déjà féru de programmation. Les mécanismes au centre d'Adventure servirent alors de socle commun pour de nombreux autres jeux qui participèrent à l'acceptation ludique de l'ordinateur personnel, comme par exemple la série des jeux Zork de l'éditeur de jeux d'aventure textuels Infocom.

D'autres jeux se sont également fondés sur ce même diagramme, mais à un coefficient moindre. Ils ont alors participé à la formation et la structuration d'autres genres en ajoutant ou en minimisant certains mécanismes. On peut à ce titre remarquer qu'en 1979, Adventure va faire l'objet d'une adaptation sur la console de salon d'Atari, la VCS, cette version souhaitant s'émanciper de la dimension textuelle d'origine pour miser davantage sur l'exploration d'un univers graphique, sacrifiant néanmoins la dimension narrative. À travers une succession de zones fixes, le joueur déplace un personnage (représenté simplement par un carré) pour trouver les trésors cachés dans les dédales d'un château. Le jeu reprend les principes d'exploration spatiale et de combinaison d'objets pour permettre la progression, mais la découverte d'une histoire ou la présence d'un récit a quasiment disparu. La présence d'ennemis à éviter ou à chasser est en revanche mise en avant, ce qui renoue avec un élément constitutif d'un des ancêtres du jeu de Crowther, Hunt the Wumpus (Yob, 1973). Les contraintes techniques de la VCS expliquent en partie cette réorientation de game design, la console se prêtant peu à l'affichage de textes du fait de sa résolution graphique, sans mentionner l'absence de clavier qui rend difficile les interactions textuelles complexes. On peut aussi ajouter que la VCS était conçue pour un contexte d'utilisation familial, qui ciblait également les enfants. Adventure de Robinett ne répond donc pas aux mêmes attentes et au même public que la version de Wood et Crowther. Le souhait de conquérir un nouveau territoire, qui se traduisit, entre autres, par l'ajout de graphismes aux jeux d'aventure, convertit l'activité d'exploration par le texte en une exploration visuelle, ce qui transforma la nature du plaisir ludique et les valeurs afférentes aux premiers jeux textuels (on s'écarte du lien qui pouvait être initialement fait entre activité ludique et programmation). 

à la tentation de prêter des caractéristiques définitives et essentialisantes à un genre de jeu, alors même que les jeux que l'on rattache à ce genre présentent des caractéristiques fluctuantes. Les multiples versions et modifications d'Adventure montrent aussi qu'un jeu est toujours susceptible d'évoluer en étant modifié par ses pratiques et qu'il est nécessaire de penser la structure de jeu non pas comme une forme fixe mais en évolution, en interaction avec l'activité ludique. Les jeux tissent aussi des liens avec d'autres types de jeux ou de logiciels, que l'on ne rattache pas nécessairement au même genre. Un même genre peut aussi être renommé ou se transformer en plusieurs autres formes qui se stabiliseront certainement pendant un temps, pour être ensuite toujours susceptibles d'évoluer à nouveau par la suite. En somme, à un moment donné, un diagramme de ludicisation peut donner forme à plusieurs genres de jeux, selon les coefficients d'actualisation.

\section{Les strates ludiques}

Cet exemple pose cependant la question de la caractérisation d'un diagramme - qui peut paraître multiforme et lui-même changeant - et de la périodisation des différentes variations effectuées par les agencements. Comme l'indique Deleuze, il y a une histoire des agencements et un devenir des mutations de diagramme (Deleuze, 1986, p. 49).

Il faut distinguer au sein du concept de diagramme plusieurs états coexistants. Il s'agit donc de tracer la carte d'un rapport de forces en fonction de ses différents mouvements immanents. Ce sont eux qui déterminent en retour la possibilité pour l'historien de périodiser (Krtolica, 2009, p. 123).

Il faut en somme repérer la constitution de "strates ludiques " et les rapports qui s'établissent entre ces strates, une strate étant chez Deleuze une "formation historique ", des « couches sédimentaires » constituées de "manière de dire et de façon de voir ", " chaque strate est faite d'une combinaison des deux, et d'une strate à l'autre, il y a variation des deux et de leur combinaison » (Deleuze, 1986, p. 56). Les contenus et la façon dont ces contenus s'expriment dans le jeu peuvent varier, faire l'objet de différentes articulations: un même jeu pouvait être vu comme un "simple" divertissement hier mais comme un outil d'apprentissage aujourd'hui, tout en disant une chose similaire (que l'on pense aux usages pédagogiques qui sont faits aujourd'hui de jeux comme Sim City). Le schéma ci-dessous permet de tenir compte de ces différentes réflexions sur le diagramme de ludicisation et les strates ludiques : 
Schéma 3. Agencements de jeu, diagramme de ludicisation et strates ludiques

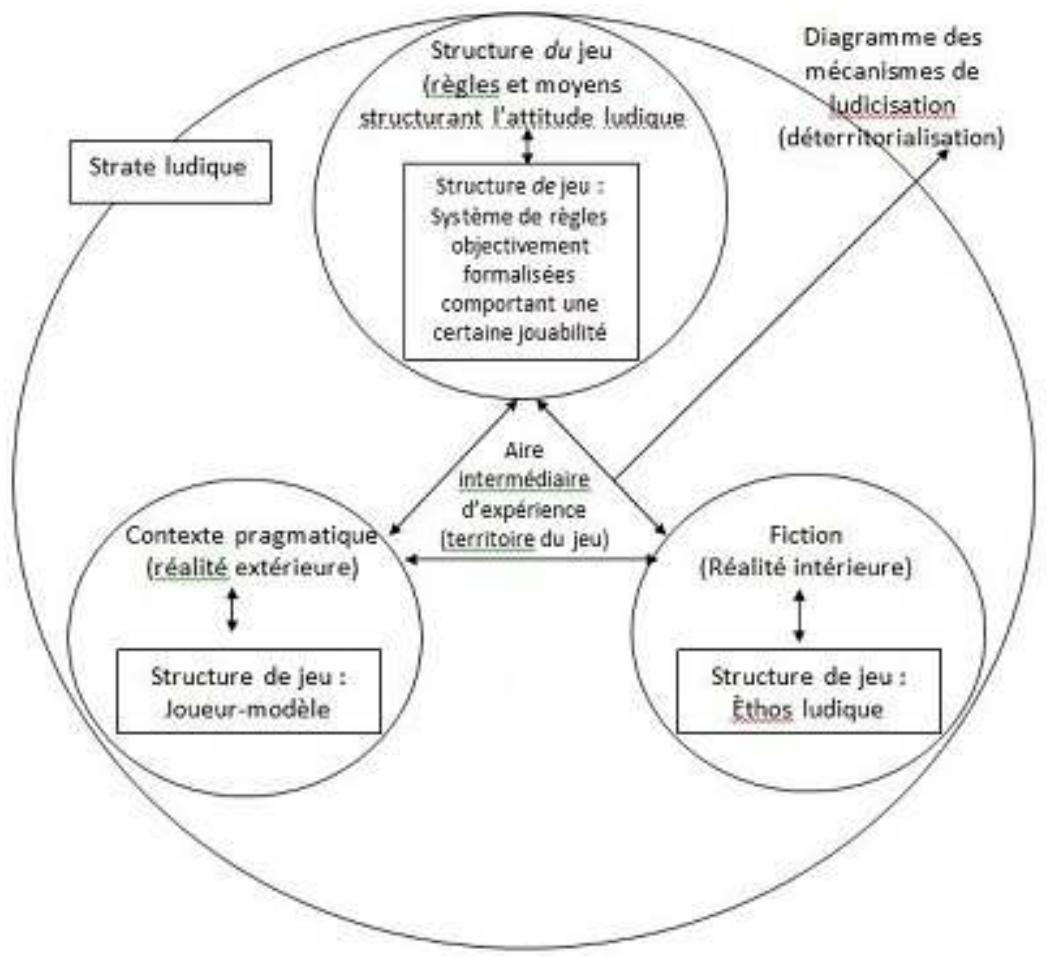

Comme l'indique Deleuze (1986, p. 56) une époque ne préexiste pas aux énoncés qui l'expriment. Il y a de la sorte des manières de dire le jeu et des façons de le voir qui peuvent varier et se combiner différemment, à charge de l'historien de périodiser ces strates et de tracer au sein de chaque strate la carte des rapports de force qui s'effectuent en fonction des mouvements du diagramme : pour les hackers des instituts de technologie américains des années 70, il y avait des façons de dire et de voir le jeu, qui étaient notamment pensées comme un modèle de libre diffusion de l'information (les jeux étaient mis en libre circulation sur l'Arpanet), il disait la fascination de ses concepteurs pour l'expérimentation technologique (jouer et programmer étaient intimement liés, comme le montre les premiers jeux d'aventure textuels). Pour les premiers promoteurs commerciaux du jeu vidéo (notamment Atari), le jeu disait l'avènement des biens expérientiels, qui se consomment dans leur usage, ils étaient donc vu comme un objet technique qui devait présenter une obsolescence rapide ; cette approche technologique de l'objet ne manqua pas de faire débat chez les premiers game designers de jeux vidéo, comme Chris Crawford, qui refusait ces considérations pour inciter à voir l' "art du game design" comme étant indépendant des critères de prouesse technologique. Pour certains artistes et théoriciens d'aujourd'hui le jeu vidéo est vu comme une nouvelle forme d'expression qui peut dire des revendications politiques, sociales, individuelles (que l'on pense au jeu September $12 t^{2}{ }^{2}$ [Newsgaming, 2003] de Gonzalo Frasca).

Comme le mentionnait Jacques Henriot :

dire que le jeu se répand chaque jour davantage, que l'on joue de plus en plus à des jeux de plus en plus nombreux, cela demeure une constatation relativement banale, qui n'atteint pas le fond du problème. Le plus important n'est pas là - mais dans le fait que l'idée même de Jeu en vienne à s'appliquer à des réalités, à des situations, à des conduites à propos desquelles son emploi, récemment encore, eût paru déplacé, voire absurde ou scandaleux. Non seulement on parle de plus en plus volontiers de 
jeu, mais aussi et surtout on en parle autrement. Nous vivons à cet égard une véritable mutation, à laquelle nous participons plus ou moins consciemment. Elle ne tient pas uniquement au fait que la pratique ludique se multiplie, se diversifie à l'extrême, mais aussi à ce que l'on en vient à qualifier de jeux ou de façons de jouer des manières d'être et de faire que l'on considérait jusque-là comme essentiellement « sérieuses » et même « dramatiques » (Henriot, 1989, p. 32).

Nous sommes entrés depuis quelques temps dans une nouvelle strate ludique. Les pistes de recherche présentées ici ont pour vocation de nous aider à voir et à dire de quelle façon les processus de ludicisation ont mené à cette « véritable mutation » à partir d'un outillage théorique approprié.

\section{Conclusion}

En 2008, nous caractérisions ainsi les spécificités de l'expérience du jeu à son ère numérique :

il y a donc à notre sens une nécessité d'analyser les jeux non pas en termes de game design mais plutôt en termes de "play design". Puisque la signification de jeu n'est pas donnée par avance mais se construit, il n'est pas possible de prendre pour acquis la dimension ludique d'un objet. L'analyse du play design revient à placer au centre de la réflexion la façon dont une expérience particulière de jeu a été modélisée dans un dispositif numérique de sorte à susciter une signification ludique partagée (Genvo, 2008).

Depuis, les réflexions orientées vers l'expérience du joueur ont acquis une certaine fortune dans la littérature académique, en France (Triclot, 2011) comme à l'étranger (voir les références mentionnées au début de l'article). Cependant, devant la multiplication des formes du ludique et au-delà de l'expérience singulière produite par chaque agencement, il nous semble à présent important de comprendre comment des significations de jeu émergent pour faire sens commun, se stabilisent et/ou évoluent. À cet effet, nous avons souhaité montrer qu'il était essentiel de considérer dans l'analyse à la fois le rôle conjoint de l'objet (game) et des pratiques (play) dans les processus de ludicisation, de façon à comprendre comment ces deux pôles composent un agencement et comment, ce faisant, le co-fonctionnement de ses éléments participe à construire et à faire évoluer les phénomènes ludiques dans leur ensemble (contenus et pratiques). En somme, pour comprendre les processus de mutation du jeu, il faut aller au-delà de la dichotomie "play studies vs. game studies ». C'est certainement le rôle d'une approche ludologique que de chercher à allier une théorisation du game et du play pour englober dans sa réflexion l'ensemble des phénomènes ludiques et leurs interactions.

\section{BIBLIOGRAPHIE}

BATESON G. (1977), Vers une écologie de l'esprit, Paris, Seuil.

BOGOST I. (2007), Persuasive games, Cambridge, MIT Press. 
BOUTAUD J.-J. (2008), « Les TIC et l'ethos », in Pignier N. (dir.), Le webdesign, Paris, Hermes Lavoisier.

CAILLOIS R. (1967), Les jeux et les hommes : le masque et le vertige [1958], Paris, Gallimard.

DELEUZE G. et PARNET C. (1977), Dialogues, Flammarion.

DELEUZE G. et GUATTARI F. (1980), Capitalisme et schizophrénie, Tome 2 : Mille plateaux, Éditions de Minuit.

DELEUZE G. (1986), Foucault, Éditions de minuit.

DUFLO C. (1997), Jouer et philosopher, Paris, Presses Universitaires de France.

FOUCAULT M. (1975), Surveiller et punir, Paris, Gallimard.

GENVO S. (2008), «Caractériser l'expérience du jeu à son ère numérique : pour une étude du "play design" ", Journée d'études Les jeux vidéo : expériences et pratiques sociales multidimensionnelles, Québec, Canada, 6 et 7 mai, disponible en ligne : http:// www.ludologique.com/wordpress/?p=105

GENVO S. (2009), Le jeu à son ère numérique. Analyser et comprendre les jeux vidéo, paris, L'Harmattan. GENVO S. (2011), «Penser les phénomènes de ludicisation du numérique : pour une théorie de la jouabilité », Revue des sciences sociales, 45, pp. 68 - 77.

HENRIOT J. (1989), Sous couleur de jouer : la métaphore ludique, Paris, José Corti.

HUIZINGA J. (1951), Homo ludens. Essai sur la fonction sociale du jeu [1938], Paris, Gallimard.

KLINE S., DYER-WITHERFORD N. \& DE PEUTER G. (2003), Digital Play. The Interaction of Technology, Culture and Marketing, Montreal \& Kingston, McGill-Queen's University Press.

KRTOLICA I. (2009), « Diagramme et agencements chez Gilles Deleuze », Filozofija I Drustvo, 3, pp. 97-123.

LETOURNEUX M. (2006), « La question du genre dans les jeux vidéo », in Genvo S. (dir.), Le game design de jeux vidéo. Approches de l'expression vidéoludique, Paris, L'Harmattan, pp. 39-54.

MALABY T. M. (2007), « Beyond Play. A new approach to games », Games and culture, vol. 2, n², pp. 95-113.

MONTFORT N. (2003), Twisty Little Passages, MIT Press.

PIGNIER N. (2008), Le webdesign, Paris, Hermes - Lavoisier.

SALEN K. \& ZIMMERMAN E. (2004), Rules of Play. Game Design Fundamentals, Cambridge, MA, MIT Press.

SCHAEFFER J.-M. (1999), Pourquoi la fiction ?, Paris, Éd. du Seuil.

SCHMOLL P. (2010), «Jeux sans fin et société ludique », in Craipeau S., Genvo S. et Simonnot B. (eds), Les jeux vidéo au croisement du social, de l'art et de la culture, Questions de communication, Série Actes, 8, pp. 27-42.

Sicart M., 2010, « Against procedurality », Game studies, vol.11, no. 3, en ligne http:// gamestudies.org/1103/articles/sicart_ap

SPERBER D. (1996), La Contagion des idées. Théorie naturaliste de la culture, Paris, Éditions Odile Jacob.

TAYLOR T.L. (2009), « The Assemblage of Play », Games and Culture, vol. 4, no. 4, pp. 331-339. 
TRICLOT M. (2011), Philosophie des jeux vidéo, Paris,Zones.

WINNICOTT D.W. (1975), Jeu et réalité, l'espace potentiel [1971], Paris, Gallimard.

\section{NOTES}

1. Spacewar est fréquemment considéré par les ouvrages d'histoire du jeu vidéo comme l'un des premiers jeux vidéo conçus. Sa conception résultait du détournement d'un des supercalculateurs du MIT. Il s'agissait pour deux joueurs de faire s'affronter deux vaisseaux spatiaux. Diffusé gratuitement sur Arpanet à travers les différents campus américains, le jeu connu de nombreuses modifications apportées par les différents joueurs.

2. Dans ce jeu, le joueur contrôle une mire de missile située au-dessus d'un village typique du Moyen-Orient. Quelques terroristes se promènent au milieu de la population. Si le joueur tente de les abattre, un énorme missile rate quasi automatiquement sa cible et fait de nombreux dommages collatéraux, qui vont générer davantage de terroristes. Il n'y a pas de moyen de gagner la partie. September 12th est une réflexion contre la politique interventionniste des ÉtatsUnis suite aux événements du 11 septembre 2001.

\section{RÉSUMÉS}

Aujourd'hui, de nombreux néologismes sont formés pour penser la contagion du jeu à différentes sphères d'activité (gamification, ludification). De même, certains théoriciens contemporains insistent sur la nécessité de considérer le jeu comme un processus mouvant (notamment Malaby), remettant en cause les conceptions essentialistes des phénomènes ludiques. Les travaux de Jacques Henriot sont à la fois précurseurs de ces nouvelles approches mais permettent également de dépasser certains clivages qui se dessinent actuellement autour de ces multiples théories sur la façon dont une situation fait jeu. Ces différentes thématiques étant au coeur de nos propres préoccupations de recherche, nous montrerons en quoi l'apport de Jacques Henriot nous a permis d'élaborer une série de notions (jouabilité, éthos ludique, ludicisation) permettant de penser les mutations des idées du jeu.

Many neologisms are formed nowadays to think the contagion of play by different spheres of activity (gamification, ludification). Likewise, contemporary theorists (such as Malaby) insist on the necessity to consider play as a changing process, questioning the essentialist conceptions of playful phenomena. Jacques Henriot is a precursor of these new approaches, but his work also allows to transcend divisions created by these multiple theories that focus on the way a situation is or is not play. These different themes being at the center of our own research interests, we will show how the work of Jacques Henriot helped us to elaborate a series of notions (playability, playful ethos, ludicisation), allowing to think the mutations of the idea of play. 
INDEX

Mots-clés : ludicisation, jouabilité, éthos ludique, jeu vidéo, agencements de jeu

Keywords : ludicisation, playability, playful ethos, video game, play agencies

\section{AUTEUR}

\section{SÉBASTIEN GENVO}

Université de Lorraine, CREM 